\title{
Tendências da hanseníase nas Mesorregiões do Pará, um Estado hiperendêmico do Norte do Brasil, 2004-2018
}

\author{
Trends of leprosy in the Mesoregions of Pará, a hyper-endemic state in Northern Brazil, \\ 2004-2018
}
Tendencias de la lepra en las mesorregiones de Pará, un estado hiperendemico del Norte de Brasil, 2004-2018

Ivone Diniz Chaquiam ${ }^{1}$, Emanuele Rocha da Silva ${ }^{1}$, José Natanael Gama dos Santos ${ }^{1}$, Belmiro Figueiredo Vinente Neto ${ }^{1}$, Joao Victor Filgueiras Mota ${ }^{1}$, Raiula Gabriela da Silva Teixeira ${ }^{1}$, Rebeca Lima Braga1, Waltair Maria Martins Pereira ${ }^{1 *}$.

\section{RESUMO}

Objetivo: Analisar a tendência da detecção da hanseníase, na população em geral, nas Mesorregiões do estado do Pará, Brasil, no período de 2004 a 2018. Métodos: Estudo ecológico exploratório utilizando o banco de dados do Sistema de Informação de Agravos de Notificação. Foram incluídos os casos de hanseníase residentes no estado de Pará. Estudadas 57.537 notificações de caso, considerando as variáveis: sexo, raça/cor da pele, idade, escolaridade, município de residência, ano da detecção da doença, classificações por forma clínica e operacional. Resultados: As maiores proporções de detecção foram observadas nas Mesorregiões Sudoeste Paraense (63,76\%), Baixo Amazonas (62,94\%), Sudeste Paraense (60,65\%) e Marajó $(60,15 \%)$. O risco para a manutenção da transmissão da doença esteve presente nas Mesorregiões Sudeste Paraense (1.476,98/100.000hab.) e Sudoeste Paraense (1.218,42/100.000hab.). A forma clínica dimorfa predominou na notificação, seguida pela forma indeterminada. A distribuição geográfica evidenciou que 2/3 do Estado apresentou área hiperendêmica, com altas taxas, envolvendo as Mesorregiões Sudeste Paraense (97,58/100 mil hab.), Sudoeste Paraense (83,19/100 mil hab.) e Marajó (84,17/100 mil hab.). Conclusão: O estudo da hanseníase no estado do Pará aponta comportamento não homogêneo e condições desfavoráveis para o controle, identificando a necessidade urgente de estratégias para tornar eficazes as ações de controle da doença.

Palavras-chave: Hanseníase, Doenças transmissíveis, Distribuição espacial.

\begin{abstract}
Objective: To analyze trends of leprosy detection in the general population in the Mesoregions Pará state, Brazil, from 2004 to 2018. Methods: Exploratory ecological study using the Information System for Notifiable Diseases database. Leprosy cases residing in the state of Pará were included. 57,537 case reports were studied, considering the variables: sex, race/skin color, age, education, municipality of residence, year of detection of the disease, classifications by clinical and operational form. Results: The highest proportions of detection were found in the Mesoregions Southwest Paraense (63.76\%), Baixo Amazonas (62.94\%), Southeast Paraense (60.65\%) and Marajó (60.15\%). The risk for maintaining the disease transmission was present in the Southeast Paraense Mesoregions (1,476.98/100,000 in hab.) and Southwest Paraense $(1,218.42 / 100,000$ in hab.). The dimorphic clinical form predominated, followed by the indeterminate form. The geographic distribution showed that $2 / 3$ of the State had a hyper-endemic area, with high rates, involving the Southeast Paraense Mesoregions (97.58/100 thousand in hab.), Southwest Paraense (83.19/100 thousand in hab.) and Marajó (84,17/100 thousand in hab.). Conclusion: The study of leprosy in Pará state points out a non-homogeneous behavior and unfavorable conditions for control, identifying the urgent need for strategies to make the disease control actions effective.
\end{abstract}

1 Universidade Federal do Pará (UFPA), Belém - PA. *E-mail: vweapaz@gmail.com 
Keywords: Leprosy, Communicable diseases, Spatial distribution.

\section{RESUMEN}

Objetivo: Analizar la tendencia de la detección de lepra en la población general de las Mesorregiones del estado de Pará, Brasil, de 2004 a 2018. Métodos: Estudio ecológico exploratorio utilizando la base de datos del Sistema de Información de Enfermedades Notificables. Se incluyeron casos de lepra residentes en el estado de Pará.Se estudiaron 57.537 reportes de casos, considerando las variables: sexo, raza / color de piel, edad, escolaridad, municipio de residencia, año de detección de la enfermedad, clasificaciones por forma clínica y operativa. Resultados: Las mayores proporciones de detección se encontraron en las Mesorregiones del Sudoeste de Paraense (63,76\%), Baixo Amazonas (62,94\%), Sureste de Paraense $(60,65 \%)$ y Marajó $(60,15 \%)$. El riesgo de mantener la transmisión de la enfermedad estuvo presente en las Mesorregiones Sureste de Paraense (1.476,98/100.000hab.) y Suroeste de Paraense (1.218,42/100.000hab.). En la notificación predominó la forma clínica dimórfica, seguida de la forma indeterminada. La distribución geográfica mostró que 2/3 del Estado tenía un área hiperendémica, con altas tasas, involucrando a las Mesorregiones Sureste Paraense (97.58/100 mil hab.), Sudoeste Paraense (83.19/100 mil hab.) Y Marajó (84,17/100 mil hab.). Conclusión: El estudio de la lepra en el estado de Pará señala comportamientos no homogéneos y condiciones desfavorables para el control, identificando la urgente necesidad de estrategias para hacer efectivas las acciones de control de la enfermedad.

Palabras clave: Lepra, Enfermedades transmisibles, Distribución espacial.

\section{INTRODUÇÃO}

A hanseníase é uma doença infectocontagiosa causada pelo bacilo intracelular obrigatório Mycobacterium lepraem, que atinge principalmente a pele e os nervos periféricos, causando sequelas dermatoneurológicas (BRASIL, 2016). Apesar da alta infectividade e baixa patogenicidade, o agente de infecção tem grande poder incapacitante devido ao acentuado poder imunogênico (CHAVES EC, et al., 2017; PIRES CAA, et al., 2015). A estreita diversidade socioeconômica torna as populações residentes em bolsões de pobrezaacentuadamente mais suscetíveis ao adoecimento por doenças negligenciadas, como a hanseníase (RIBEIRO MDA, et al., 2018; MONTEIRO LD, et al., 2015).

A primeira sistematização da especificação das formas da doença, ocorrida em 1953 pela classificação de Madri, agregou critérios de polaridade a partir de aspectos clínicos e bacteriológicos, ordenando as formas clínicas em grupos polares - tuberculóide $(\mathrm{T})$, Virchowiano $(\mathrm{V})$ e lepromatoso $(\mathrm{L})$-, grupo transitório - forma indeterminada (I) - e grupo instável - forma dimorfa (D) ou borderline (B) (BASOMBRIO B, et al., 1953).

A Organização Mundial da Saúde (OMS), no ano de 1988 preconizou o uso de classificação operacional, para auxiliar na alocação dos indivíduos nos esquemas de tratamento específico, principalmente nos locais de maiores dificuldades de apoio por diagnóstico laboratorial, definindo então os casos de acordo com critérios clínicos, priorizando a história clínica, epidemiológica e o exame dermatoneurológico. Esta classificação se divide em duas formas, a paucibacilar (PB) quando o paciente apresenta até cinco manchas ou lesões cutâneas e a multibacilar (MB), quando apresenta mais de cinco manchas ou lesões cutâneas (BRASIL, 2016).

Seu diagnóstico é essencialmente clínico, através do exame dermatoneurológico, podendo ser complementado pela baciloscopia de raspado dérmico para a identificação do índice e da morfologia bacilar. Quando positivo, o caso deverá ser classificado como MB, independentemente do número de manchas ou lesões na pele (BRASIL, 2016). Além disso, a hanseníase é uma doença curável, cujo tratamento medicamentoso é gratuito e oferecido, no Brasil, pelo Sistema Único de Saúde (SUS), consistindo em um esquema de poliquimioterapia (PQT) (BRASIL, 2016).

A implementação da PQT foi essencial para a redução da carga da doença no mundo, entretanto, por diversos condicionantes, o Brasil apresenta dificuldades para a execução das atividades de controle da hanseníase. Nesse sentido, acordos Internacionais foram assinados, o último, para o período de 2016 a 2020 , está correlacionado ao Plano de Ação Para a Eliminação de Doenças Infecciosas Negligenciadas e Ações 
Pós-Eliminação. Esse Plano aborda, principalmente, a vigilância, o manejo, o controle e a eliminação da hanseníase e outras 12 doenças selecionadas. Propõe atividades atualizadas, claras e inovadoras, para reduzir a morbidade e as incapacidades físicas, além de abordar estratégias para o combate ao estigma. $O$ objetivo do Plano é alcançar a condição de eliminação da hanseníase em consonância com os objetivos de Desenvolvimento Sustentável da Organização das Nações Unidas (ONU) até 2030 (ONU, 2015).

No nível mundial, em 2018, foram notificados 208.619 casos novos de hanseníase com taxa de detecção de 2,74 casos por 100.000 habitantes. Os três países com as mais altas cargas da doença, responsáveis por $79,6 \%$ dos casos novos do mundo, foram à Índia com 120.334, o Brasil com 28.660 e a Indonésia com 17.017 (OMS, 2018).

O Brasil está entre os 22 países que apresentaram as mais altas cargas mundiais de hanseníase e ocupa a segunda posição mundial em relação à detecção de casos novos. No ano de 2018 , notificou 28.660 casos novos, com taxa de detecção na população em geral de 13,70 casos por cada 100 mil habitantes e menores de 15 anos de idade 3,75 casos para cada 100.000. A taxa de grau ll de incapacidade física, no momento do diagnóstico, foi de 10,08/ 1 milhão de habitantes (BRASIL, 2020).

A distribuição da doença no país não ocorre de forma homogênea e seguramente é influenciada por determinantes socioeconômicos existentes em cada Unidade Federada. As áreas de maior concentração de casos localizam-se nas regiões Centro-Oeste, Norte e Nordeste do país, com os estados de Mato Grosso, Maranhão, Tocantins e Pará liderando as maiores notificações de casos (RODRIGUES RN, et al., 2020).

O objetivo deste trabalho foi analisar a tendência da detecção da hanseníase nas seis Mesorregiões do estado do Pará, no período de 2004 a 2018.

\section{MÉTODOS}

Foi realizado um estudo ecológico sobre a ocorrência de casos novos de hanseníase, no Pará. O Estado apresenta área territorial de $8.664,50 \mathrm{~km}^{2}$, com população estimada em 8.578 .051 habitantes, no ano de 2018, com 6,87 habitantes por quilômetro quadrado. É composto por 144 municípios, aglutinados em seis Mesorregiões formadas por municípios com características geográficas e socioeconômicas similares, apenas para fins estatísticos não constituindo uma entidade política ou administrativa (IBGE, 2012).

A fonte dos dados foi o Sistema de Informação de Agravos de Notificação (SINAN) gerenciado pela Secretaria de Saúde Pública do Pará (SESPA), referente aos casos novos de hanseníase notificados/investigados pelos municípios, no período de 2004 a 2018 (SESPA, 2019).

Foram incluídos os registros de todos os casos notificados como hanseníase residentes no Pará e excluídos aqueles cujo diagnóstico da doença não foi confirmado e aqueles que não dispunham, no banco de dados, de informações para a categorização de variáveis, em tabela de contingência, o que determinou para esta análise especifica a exclusão de 4.512 casos.

Para a variável raça/cor da pele, preto e pardo foram aglutinadas, considerando que as categorias utilizadas pelo SINAN são as mesmas utilizadas pelo Instituto Brasileiro de Geografia e Estatística (IBGE), que, no Estatuto da Igualdade Racial (Lei $\left.n^{\circ} 12.288 / 2010\right)$, define a população negra com o "conjunto de pessoas que se autodeclararam pretas e pardas" (IBGE, 2014). A descrição do perfil sociodemográfico foi realizada a partir das variáveis sexo, raça/cor da pele, idade, escolaridade e município de residência, constantes na ficha de notificação/investigação de caso. A análise dos aspectos clínico-epidemiológicos tomou por base as variáveis ano da detecção do caso, as classificações das formas clínicas e a operacional da doença.

As taxas de detecção foram ajustadas, pela idade, tanto para os municípios como para as Mesorregiões, não tendo sido encontradas diferenças significantes na distribuição proporcional das diferentes áreas populacionais, ao longo da série histórica analisada. 
Os parâmetros para a classificação de endemicidade da doença foram aqueles recomendados pelo Ministério da Saúde (MS) que se expressam em baixa, média, alta, muito alta endemicidade e hiperendêmico (BRASIL, 2016).

As unidades de análise foram as Mesorregiões, disponibilizadas pelo IBGE, assim especificadas com o número de municípios a cada uma delas agregados: Baixo Amazonas (15), Marajó (16), Metropolitana de Belém (11), Nordeste Paraense (49), Sudoeste Paraense (14), Sudeste Paraense (39) (IBGE, 2012).

Buscando minimizar possíveis variações sazonais e temporais, da ocorrência de casos e das populações, que pudessem interferir no cálculo das taxas de detecção da hanseníase na população geral, com vistas à observação da redução proporcional no decurso do período estudado, os dados foram agrupados em intervalos trimestrais distintos.

Os dados foram apresentados sob a forma de tabelas, gráfico e mapa. Foram aplicados a estatística descritiva e o teste qui-quadrado de aderência para a análise da distribuição de frequências, utilizando o programa BioEstat v5.3, e consideradas como diferenças estatisticamente significantes aquelas com $p<0,05$ (AYRES, et. al., 2019). A distribuição geográfica foi realizada no software livre com código-fonte aberto QGis v2.18, e representada através de mapa temático.

O projeto deste estudo faz parte do Programa de Apoio ao Doutor Pesquisador (PRODOUTOR) aprovado na Universidade Federal do Pará (UFPA). O estudo foi realizado atendendo as determinações Resolução $n^{\circ}$ 466 de 12 de dezembro de 2012, do Conselho Nacional de Saúde (CNS) que estabelece diretrizes e normas regulamentadores de pesquisas envolvendo seres humanos. Foi aprovado pelo Comite de Ética e Pesquisa (CEP) do Instituto de Ciências da Saúde (ICS) da UFPA, com Certificado de Apresentação para Apreciação (CAAE) n³3158219.5.0000.0018; Parecer $n^{\circ}$ 4.146.997, de 09 de julho de 2020.

\section{RESULTADOS}

Entre 2004 e 2018, no Pará, foram notificados 57.537 novos casos de hanseníase, com média anual de 3.836 casos notificados. A ocorrência em menores de 15 anos de idade foi de 11,18\% (6.431/57.537).

O perfil demográfico da hanseníase, para todas as Mesorregiões, consistiu em predomínio de indivíduos do gênero masculino. A média de idade para os menores de 15 anos foi de $10,56( \pm 1)$, para os de 20 a 39 anos foi de $29,46( \pm 1)$ e para a faixa etária de 40 a 59 anos foi de $48,60( \pm 1)$. As médias de idade para menores de 15 anos, em todas as Mesorregiões, foram superiores à do Estado, a especificar Nordeste Paraense, Metropolitana de Belém, Sudeste, Sudoeste, Marajó e Baixo Amazonas, com 10,75 ( \pm 3$), 10,63$ ( \pm 3$), 10,75( \pm 2), 10,69( \pm 4), 10,66( \pm 3)$ e $10,99( \pm 7)$, respectivamente.

Para a faixa etária de 20 a 39 anos, a média da idade das Mesorregiões Nordeste Paraense $(29,17 \pm 1)$ e Sudeste Paraense $(29,17 \pm 1)$ e para a faixa etária de 40 a 59 anos, as Mesorregiões Nordeste Paraense $(40,04 \pm 1)$ e Sudeste Paraense $(40,04 \pm 1)$, ficaram abaixo da média de idade do Estado.

A variável cor da pele parda e/ou negra, em relação às demais, apresentou significância estatística $(p<0,001)$ nas seis Mesorregiões. As Mesorregiões Nordeste Paraense $(84,64 \%)$, Marajó $(85,76 \%)$ e Baixo Amazonas (84,84\%), apresentaram proporções maiores que a do Estado $(80,78 \%)$. A escolaridade dos casos novos notificados em todas as Mesorregiões registrou a maior proporção para o Ensino Fundamental Incompleto.

As Mesorregiões Sudeste Paraense (42,54\%) e Metropolitana de Belém (20,43\%) foram as que apresentaram as maiores proporções de notificação de casos novos de hanseníase, contudo o maior risco para a manutenção da transmissão da doença foi observado pra as Mesorregiões Sudeste (1.476,98/100.000hab.) e Sudoeste (1.218,42/100.000hab.) (Tabela 1). 
Tabela 1 - Casos novos e taxas de detecção geral cumulativa de hanseníase, segundo Mesorregiões do estado do Pará, Brasil, 2004 a 2018.

\begin{tabular}{ccccc}
\hline Mesorregiões & Casos novos & $\%$ & População* & Taxa de Detecção \\
\hline Baixo Amazonas & 2.118 & 3,68 & 2.746 .958 & 283,55 \\
Marajó & 2.359 & 4,10 & 488.698 & 482,71 \\
Metropolitana de Belém & 11.753 & 20,43 & 2.496 .414 & 470,80 \\
Nordeste Paraense & 10.594 & 18,41 & 1.798 .037 & 589,30 \\
Sudoeste Paraense & 6.236 & 10,84 & 511.809 & 1218,42 \\
Sudeste Paraense & 23.477 & 10,84 & 1.657 .230 & 1476,98 \\
\hline Total do Estado & $\mathbf{5 7 . 5 3 7}$ & $\mathbf{1 0 0 , 0 0}$ & $\mathbf{7 . 6 9 9 . 1 4 6}$ & $\mathbf{7 4 7 , 3 2}$
\end{tabular}

Legenda: * População média para o período. † Por 100.000 habitantes. Fonte: Chaquiam ID, et al., 2021; dados extraídos do SINAN, 2019.

Quando comparados o primeiro e o último triênio do estudo, a maior redução da taxa de detecção de hanseníase, na população em geral, ocorreu na Mesorregião Sudeste Paraense $(67,44 \%)$, sendo esta redução maior que a do estado do Pará $(61,63 \%)$ (Tabela 2).

Tabela 2 - Redução das taxas de detecção de hanseniase, na população em geral, entre os triênios 20042006 e 2016-2018, segundo Mesorregiões do estado do Pará, Brasil.

\begin{tabular}{ccccc}
\hline Mesorregiões & $\mathbf{2 0 0 4 - 2 0 0 6}$ & $\mathbf{2 0 1 6} \mathbf{- 2 0 1 8}$ & $\mathbf{2 0 1 8 - 2 0 0 4}$ & Redução de Detecção \% \\
\hline Baixo Amazonas & 28,78 & 12,16 & 16,62 & 57,74 \\
Marajó & 32,54 & 20,14 & 12,40 & 38,10 \\
Metropolitana de Belém & 59,54 & 23,40 & 36,13 & 60,69 \\
Nordeste Paraense & 49,43 & 22,73 & 26,71 & 54,03 \\
Sudoeste Paraense & 123,00 & 51,02 & 71,98 & 58,52 \\
Sudeste Paraense & 159,21 & 51,83 & 107,37 & 67,44 \\
\hline Total do Estado & $\mathbf{8 3 , 4 4}$ & $\mathbf{3 2 , 0 2}$ & $\mathbf{5 1 , 4 2}$ & $\mathbf{6 1 , 6 3}$ \\
\hline
\end{tabular}

Fonte: Chaquiam ID, et al., 2021; dados extraídos do SINAN, 2019.

Em todas as Mesorregiões, a forma clínica dimorfa foi a de maior proporção de notificação, com diferença estatisticamente significante entre as demais formas $(p<0,001)$. Nas Mesorregiões Sudeste Paraense $(46,96 \%)$ e Sudoeste Paraense $(46,85 \%)$, a frequência de notificação de casos da forma dimorfa foi superior à frequência observada para o Estado (43,87\%). A segunda forma clínica de maior prevalência foi a indeterminada, que apresentou proporções superiores à observada para o Estado (22,84\%) nas Mesorregiões Sudeste Paraense (25,44\%), Sudoeste Paraense (28,47\%) e Marajó (23,05\%). A Mesorregião Metropolitana de Belém apresentou a segunda forma clínica de maior prevalência a tuberculóide (22,81\%) (Tabela 3).

Para o Estado, a frequência dos casos das formas clínicas dimorfa e Virchowiana representou $57,59 \%$ do total das notificações, sendo que apenas a Mesorregião Nordeste Paraense $(54,44 \%)$, apresentou proporção abaixo da do Estado (Tabela 3).

Os casos MB foram as mais prevalentes em todas as Mesorregiões, sendo que quatro delas, Sudoeste Paraense (58,17\%), metropolitana de Belém (61,23\%) Baixo Amazonas (63,10\%) e Marajó (59,70\%) apresentaram frequências superiores à do Estado (57,53\%). Para os casos PB, apenas duas Mesorregiões, Sudeste Paraense $(43,77 \%)$ e Nordeste $(45,55 \%)$ apresentaram frequências maiores que a do Estado (42,47\%) (Tabela 3).

A tendência da detecção da Mesorregião Sudeste Paraense, mesmo em decréscimo, esteve em proporções muito acima da do Estado. As demais Mesorregiões apresentaram as taxas de detecção em proporções de regressão abaixo da do Estado (Figuras 1a e 1b). 
Tabela 3 - Distribuição dos casos novos de hanseníase, segundo a forma clínica e a classificação operacional, por Mesorregiões do estado do Pará, Brasil, 2004 a 2018.

\begin{tabular}{|c|c|c|c|c|c|c|}
\hline \multirow{2}{*}{ Mesorregiões } & \multirow{2}{*}{ Forma Clínica* } & \multicolumn{2}{|c|}{ Classificação Operacional** } & \multirow{2}{*}{ Total } & \multirow{2}{*}{$\%$} & \multirow{2}{*}{ p-valor } \\
\hline & & PB & MB & & & \\
\hline \multirow{7}{*}{ Baixo Amazonas } & Indeterminada & 416 & - & 416 & 21,71 & - \\
\hline & Tuberculóide & 248 & - & 248 & 12,94 & - \\
\hline & Dimorfa & - & 783 & 783 & 40,87 & $<0,0001$ \\
\hline & Virchowiana & - & 426 & 426 & 22,23 & - \\
\hline & Não classificado & 43 & - & 43 & 2,24 & - \\
\hline & Total & 707 & 1209 & 1916 & 100 & - \\
\hline & $\%$ & 36,9 & 63,1 & - & 100 & $<0,0001$ \\
\hline \multirow{7}{*}{ Marajó } & Indeterminada & 310 & - & 310 & 23,05 & - \\
\hline & Tuberculóide & 169 & - & 169 & 12,57 & - \\
\hline & Dimorfa & - & 544 & 544 & 40,45 & $<0,0001$ \\
\hline & Virchowiana & - & 259 & 259 & 19,26 & - \\
\hline & Não classificado & 63 & - & 63 & 4,68 & - \\
\hline & Total & 542 & 803 & 1345 & 100 & - \\
\hline & $\%$ & 40,3 & 59,7 & - & 100 & $<0,0001$ \\
\hline \multirow{7}{*}{$\begin{array}{c}\text { Metropolitana de } \\
\text { Belém }\end{array}$} & Indeterminada & 1728 & - & 1762 & 15,35 & - \\
\hline & Tuberculóide & 2533 & - & 2619 & 22,81 & - \\
\hline & Dimorfa & - & 4819 & 4843 & 42,18 & $<0,0001$ \\
\hline & Virchowiana & - & 2211 & 2221 & 19,35 & - \\
\hline & Não classificado & 190 & - & 190 & 1,65 & - \\
\hline & Total & 4451 & 7030 & 11481 & 100 & - \\
\hline & $\%$ & 38,77 & 61,23 & - & 100 & $<0,0001$ \\
\hline \multirow{7}{*}{ Nordeste Paraense } & Indeterminada & 2198 & - & 2198 & 22,46 & - \\
\hline & Tuberculóide & 1396 & - & 1396 & 14,27 & - \\
\hline & Dimorfa & - & 3717 & 3717 & 37,98 & $<0,0001$ \\
\hline & Virchowiana & - & 1611 & 1611 & 16,46 & - \\
\hline & Não classificado & 864 & - & 864 & 8,83 & - \\
\hline & Total & 4458 & 5328 & 9786 & 100 & - \\
\hline & $\%$ & 45,55 & 54,45 & - & 100 & $<0,0001$ \\
\hline \multirow{7}{*}{ Sudoeste Paraense } & Indeterminada & 1638 & - & 1638 & 28,47 & - \\
\hline & Tuberculóide & 572 & - & 572 & 9,94 & - \\
\hline & Dimorfa & - & 2696 & 2696 & 46,85 & $<0,0001$ \\
\hline & Virchowiana & - & 651 & 651 & 11,31 & - \\
\hline & Não classificado & 197 & - & 197 & 3,42 & - \\
\hline & Total & 2407 & 3347 & 5754 & 100 & - \\
\hline & $\%$ & 41,83 & 58,17 & - & 100 & $<0,0001$ \\
\hline \multirow{7}{*}{ Sudeste Paraense } & Indeterminada & 5786 & - & 5786 & 22,44 & - \\
\hline & Tuberculóide & 3430 & - & 3430 & 15,08 & - \\
\hline & Dimorfa & - & 10680 & 10680 & 46,96 & $<0,0001$ \\
\hline & Virchowiana & - & 2109 & 2109 & 9,27 & - \\
\hline & Não classificado & 738 & & 738 & 3,24 & - \\
\hline & Total & 9954 & 12789 & 22743 & 100 & - \\
\hline & $\%$ & 43,77 & 56,23 & - & 100 & $<0,0001$ \\
\hline \multirow{7}{*}{ Estado do Pará } & Indeterminada & 12076 & - & 12110 & 22,84 & - \\
\hline & Tuberculóide & 8348 & - & 8434 & 15,91 & - \\
\hline & Dimorfa & - & 23239 & 23263 & 43,87 & $<0,0001$ \\
\hline & Virchowiana & - & 7267 & 7277 & 13,72 & - \\
\hline & Não classificado & 2095 & - & 2095 & 3,95 & - \\
\hline & Total & 22519 & 30506 & 53025 & 100 & - \\
\hline & $\%$ & 42,47 & 57,53 & & 100 & 0 \\
\hline
\end{tabular}

Legenda: *Foram excluídos os casos que apresentaram erro nas formas clínicas versus classificação operacional. ${ }^{* *}$ Foram excluídos os casos que apresentaram erro na classificação operacional versus formas clínicas. Fonte: Chaquiam ID, et al., 2021; dados extraídos do SINAN, 2019. 
Figura 1 - Tendência da taxa de detecção da hanseníase, na população em geral, média por triênios, por Mesorregião do Estado do Pará, Brasil, 2004 a 2018.

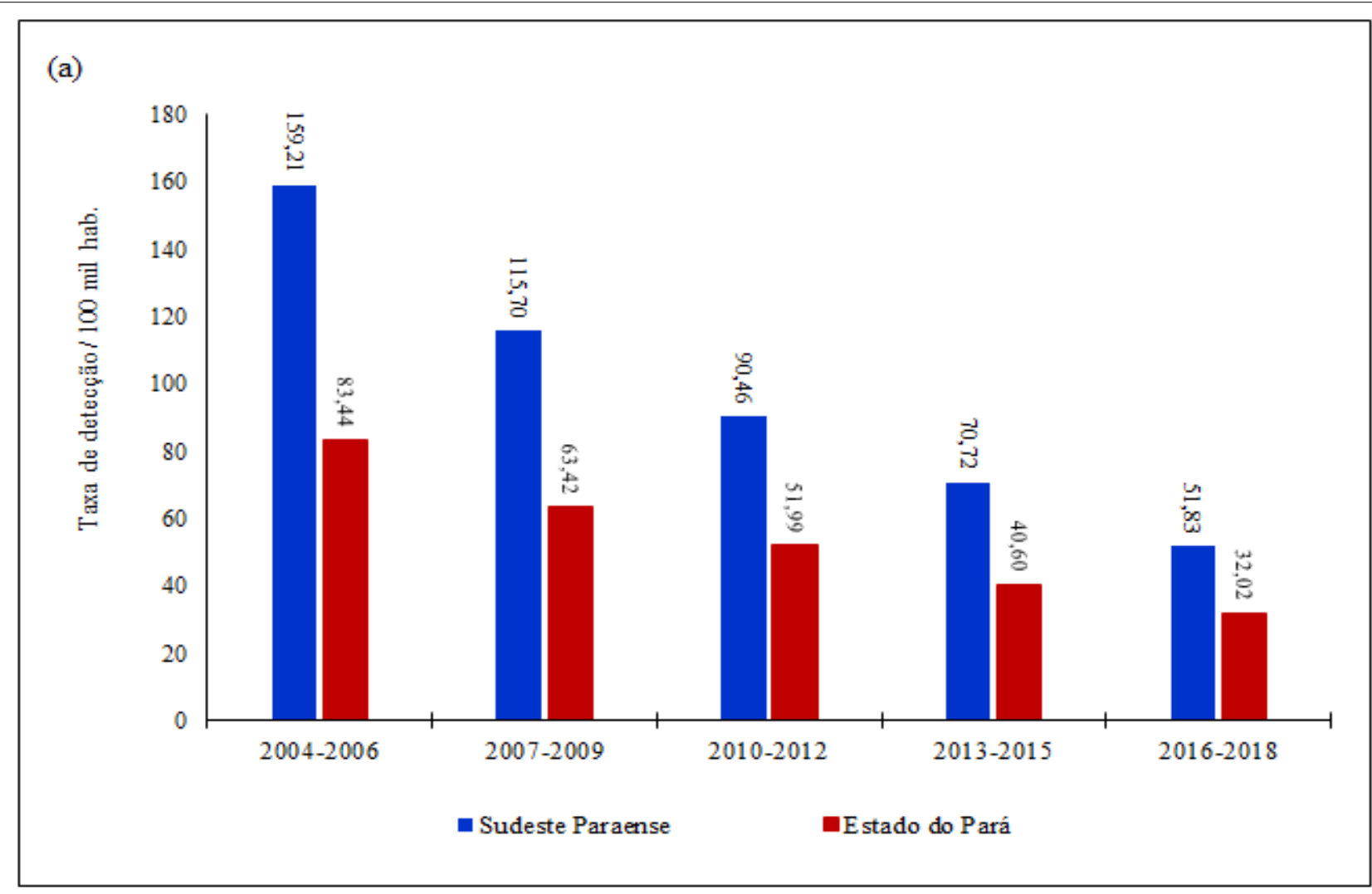

(b)

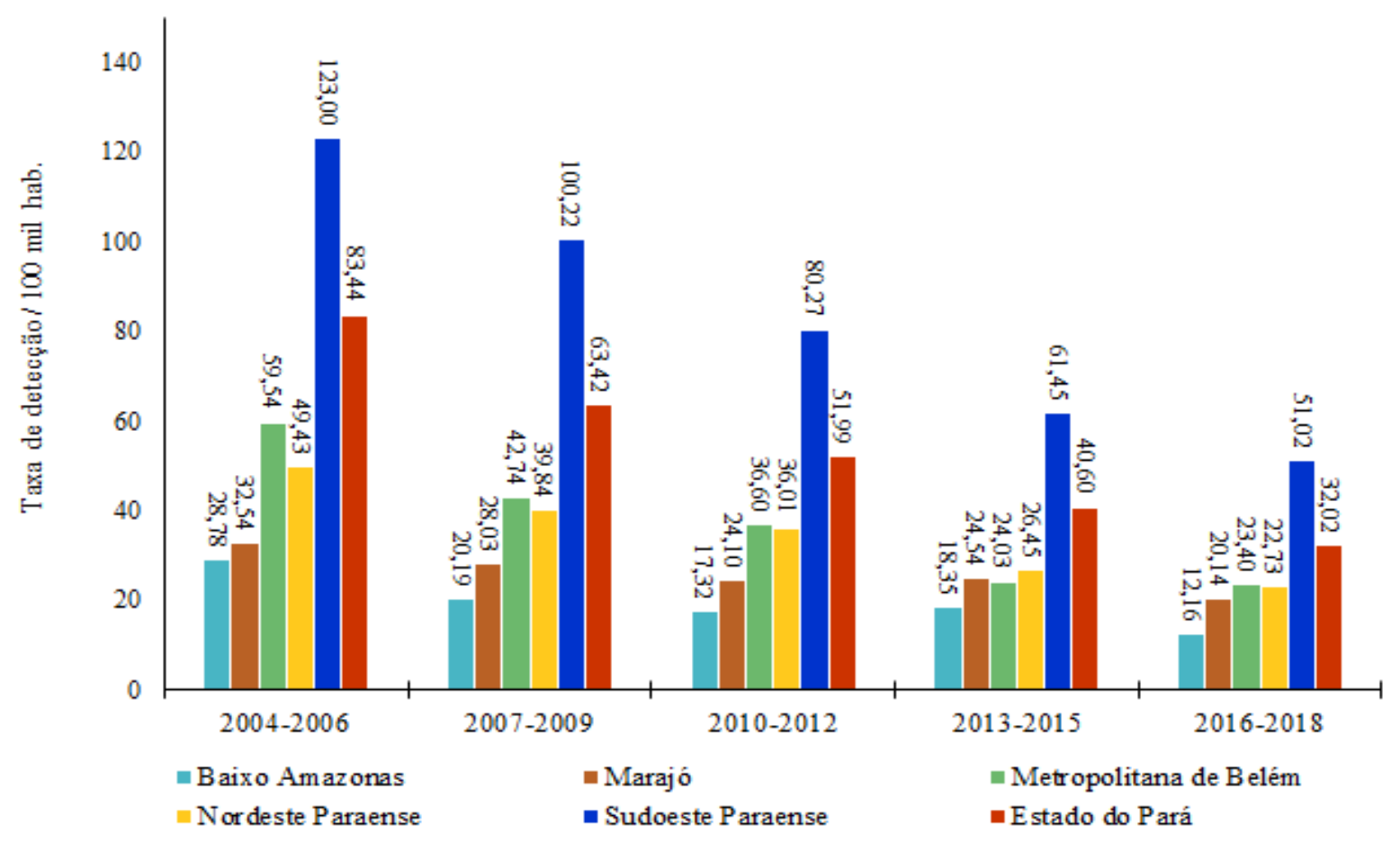

Legenda: a) Mesorregião com valor de redução da taxa de detecção da hanseníase acima da redução do Estado. b) Mesorregiões com valores de redução da taxa de detecção da hanseníase abaixo da redução do Estado. Fonte: Chaquiam ID, et al., 2021; dados extraídos do SINAN, 2019. 
A distribuição geográfica das taxas médias de detecção da hanseníase, na população em geral, permite visualizar que 2/3 do Estado como área hiperendêmica, envolvendo as Mesorregiões Sudeste Paraense, Sudoeste Paraense e Marajó, com altas taxas de detecção da hanseníase. A Mesorregião do Baixo Amazonas foi a que apresentou a menor taxa média de detecção (29,52/100 mil hab.) classificando-se como área de muito alta endemicidade (Figura 2).

Figura 2 - Distribuição geográfica das taxas médias de detecção da hanseníase, na população em geral, por Mesorregiões do Estado do Pará, Brasil, 2004 a 2018.

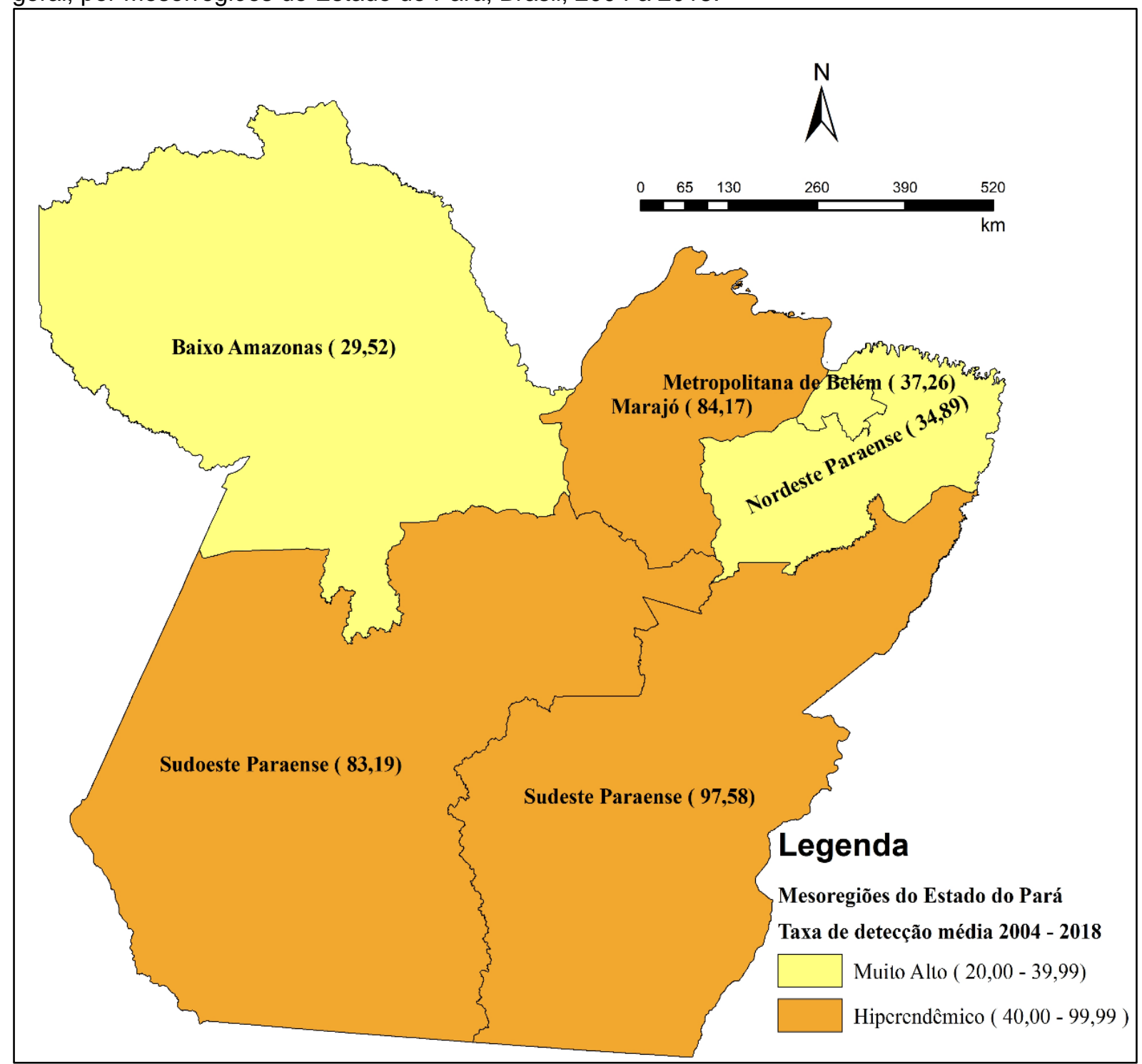

Fonte: Chaquiam ID, et al., 2021; dados extraídos do SINAN, 2019.

\section{DISCUSSÃO}

A maior proporção de casos de hanseníase no sexo masculino não difere do que já está explicitado na literatura disponível (OLIVEIRA AEVM, et al., 2020; QUARESMA MSM, et al., 2019; STIVANIN JJ, et al., 2020). Embora a proporção de casos seja maior nos homens, não existe comprovação de que a mulher apresente fatores de proteção (WHO, 2017). Possivelmente os homens tenham menos cuidados com a aparência de seus corpos, procurem menos e apresentem dificuldade de acesso aos serviços de saúde devido a não disponibilidade destes nos finais de semanas e feriados, o que os incompatibiliza de buscar assistência devido a carga laboral exercida no mesmo horário em que as unidades de saúde estão em funcionamento. No Brasil, a população feminina dispõe de programas especiais para o tratamento de sua 
saúde, assim como têm mais oportunidades de frequentar as unidades de saúde para o acompanhamento da saúde dos filhos. Essa caracterização indica diferenças de acesso ao programa de controle da doença e a dinâmica da população para utilizar os serviços em prol de suas necessidades (BRASIL, 2020).

Houve predomínio de indivíduos diagnosticados como casos novos de hanseníase que declararam ter cor da pele preta e/ou parda em todas as Mesorregiões, concordando com os achados por ocasião do censo de 2010, que identificou no Pará a frequência de $76,5 \%$ de pessoas que se autodeclararam de cor de pele negra e/ ou parda (IBGE, 2012). Este achado permite voltar o olhar para as desigualdades existentes e refletir que esse segmento populacional, que historicamente representa a maior parte da população do país, é o que mais sofre com as desigualdades advindas da vida social e econômica da estrutura social a que pertençam, incluindo, inclusive, as dificuldades para o acesso ao sistema de saúde (ABRITA APCT e ABRITA MB, 2018; PASSOS ALV e ARAÚJO LF, 2020).

As maiores frequências para a cor da pele preta e/ou parda foram observadas nas Mesorregiões Nordeste (84,64\%), Marajó (85,76\%) e Baixo Amazonas (84,84\%). Essas Mesorregiões são as tem os menores números de projetos de exploração mineral, de desenvolvimento do agronegócio e agropecuária no Estado, principalmente dos municípios que fazem fronteira com o Pará (DA SILVA JM, 2016).

A predominância de casos nas faixas etárias de 20 a 39 anos e de 40 a 59 anos de idade em todas as Mesorregiões sinaliza para o maior segmento da população, que é de pessoas jovens e de adultos, que participam de grupos de maior movimentação nos territórios em busca de trabalho e renda. Esse fato levanta a possibilidade da afirmativa de que a hanseníase pode ser considerada uma doença do adulto jovem, que compõe a faixa etária economicamente ativa, fato que pode determinar prejuízos no que tange a economia do Estado, devido às possibilidades de licenças à saúde, bem como a facilidade para a transmissão da doença, devido essa faixa etária ser constituída por pessoas de maior autonomia de circulação e maior participação em aglomerados populacionais sociais e de trabalho (ARAÚJO MM, 2015). Quando ocorre um acréscimo de casos detectados nessas faixas etárias, as evidências apontam que existe um aumento na cadeia de transmissão do bacilo na população em geral, além de uma possível deficiência na vigilância e no controle da doença (MONTEIRO LD, et al., 2015).

Os achados sobre a escolaridade ratificam o que já se encontra disposto na literatura, em que se observa uma predominância em indivíduos com baixos níveis de escolaridade, traduzindo que os fatores sociais têm forte relação com a determinação da doença (QUARESMA MSM, et al., 2019; CUNHA MHCM, et al., 2017). Esses achados são importantes para o desenvolvimento de atividades de educação em saúde para a população, bem como para facilitar a apreensão das orientações sobre o tratamento e o auto cuidado, necessários no decurso do tratamento e acompanhamento (COSTA LA, et al., 2017).

O indivíduo com hanseníase passa pelo processo de autorejeição, pelo fato de essa ser, há muito, estigmatizadora; isso dificulta a aceitação do diagnóstico e consequentemente o tratamento e acompanhamento, que são longos (PASSOS ALV e ARAÚJO LF, 2020; ABRITA APCT e ABRITA MB, 2018). Esse fato acaba sendo determinante para que ocorra recusa ou abandono de tratamento, fatores que precisam ser monitorados pelas equipes que desenvolvem a Atenção Primária da Saúde (APS).

A análise da evolução das taxas de detecção de hanseníase, aglutinadas por triênio, aponta regressão no decurso da série estudada em todas as Mesorregiões do Estado. Três delas, Sudoeste Paraense (83,19/100 mil hab.), Sudeste (97,58/100 mil hab.) e Marajó (84,17/100 mil hab.), apresentaram taxas de detecção altas e se classificam como áreas hiperendêmicas, definindo a maior de risco para a transmissão da doença. As Mesorregiões Nordeste (34,89/100 mil hab.), Metropolitana de Belém (37,26/100 mil hab.), e Baixo Amazonas (29,52/100 mil hab.), apresentaram decréscimo, entretanto se mantém como áreas de muito alta endemicidade. $O$ padrão observado nas Mesorregiões do Pará não difere do encontrado em pesquisas realizadas em outros estados da Região Norte, território do país que concentra as maiores notificações de casos novos de hanseníase (BRASIL 2020; RODRIGUES RN, et al.,2020; RIBEIRO MDA, et al., 2018; MONTEIRO LD, et al., 2015). 
A maior proporção de casos diagnosticados na forma multibacilar, especificamente a dimorfa, que é a forma clínica que tem um grande poder de transmissibilidade e uma alta possibilidade de causar incapacidades físicas residuais, é um forte indicativo que o diagnóstico está ocorrendo de forma tardia, assim como corrobora com a discussão de que a cadeia de transmissão do bacilo continua a ocorrer e representa o farol que ilumina para o alerta da necessidade de implementação das atividades de vigilância da doença, por serem estas formas consideradas as contagiantes da doença e potencialmente as mais incapacitantes, e não só com busca ativa de casos suspeitos, mas com atividades de prevenção instituídas (COSTA LA, et al., 2017; SANTOS DS, et al., 2018).

A frequência de diagnóstico em formas PB nas Mesorregiões Sudeste $(43,77 \%)$ e Nordeste Paraenses $(45,55 \%)$ acima da proporção do Estado $(42,47 \%)$ pode sinalizar para a melhoria da vigilância da doença centrada na busca ativa de casos. A literatura referência que principalmente os contatos domiciliares têm maior risco para o adoecimento, sendo 3,8 vezes maior o risco para os contatos das formas MB (LOBATO DC, et al., 2016).

A regressão da tendência de detecção observada pode ser considerada como uma situação ilusória e não um indicativo de controle da doença, uma vez que são altas as médias das taxas de detecção por Mesorregiões e cerca de 2/3 do Estado envolvendo duas das Mesorregiões (Sudoeste Paraense, Sudeste Paraense) que tem seus acessos facilitados pela migração estimulada pelos processos de extração mineral, implementação de agropecuária e a construção de Hidroelétrica e é favorecido pela rede rodoviária que liga o Pará internamente, mas também com os Estado de Mato Grosso, Maranhão e Tocantins (DA SILVA JM, 2016; PEREIRA WMM, 2018).

A Mesorregião do Marajó, com taxa média de detecção alta e território hiperendêmico, engloba $6 \%$ da população do Estado e apresenta a menor organização populacional quando comparada às outras Mesorregiões, com dificuldade de acesso - o qual na maioria das vezes se faz pelos rios da Amazônia -, e congrega os municípios que apresentam os menores índices de escolaridade da população paraense, agregando dessa forma múltiplos fatores de risco para a manutenção da hanseníase (PEREIRA, WMM, 2018).

\section{CONCLUSÃO}

O estudo da situação epidemiológica da hanseníase nas Mesorregiões do Estado do Pará forneceu informações sobre o padrão heterogêneo da sua distribuição geográfica, com a identificação de áreas de alta endemicidade. Possibilita a adequação do planejamento que envolva providências urgentes como a disponibilidade de serviços de APS para maior cobertura populacional, capacitação das equipes para a execução de trabalho adequado de vigilância e acompanhamento dos casos detectados da doença visando o efetivo controle. O quadro epidemiológico observado sugere pesquisas com novas abordagens metodológicas para a compreensão dos fatores que condicionam a manutenção de altas taxas de detecção no Pará.

\section{REFERÊNCIAS}

1. ABRITA APCT, ABRITA MB. Hanseníase: aspectos psicológicos e socioeconômicos. Brazilian Journal of Scientific Administration, 2018; 9(1): 81-92.

2. ARAÚJO MM, et al. Perfil Clínico-Epidemiológico De Pacientes Que Abandonaram o Tratamento de Hanseníase. Hansen Int, 2015; 39 (2): 55-63.

3. AYRES M, et al. BioEstat 5.3: aplicações estatísticas nas áreas das ciências biológicas e médicas. Belém: Sociedade Mamirauá, 2019.

4. BASOMBRIO B, et al. Commision of classification - Draft Report of Classification Committee. Memória de VI Congresso Internacional de Leprologia. Madrid: Gobierno de España y Asociacion Internacional de la Lepra, 1953: 75-86.

5. BRASIL. Ministério da Saúde. Secretaria de Vigilância em Saúde. Departamento de Doenças de Condições Crônicas e Infecções Sexualmente Transmissíveis. Boletim Epidemiológico de Hanseníase. Número Especial/jan. 2020. 
6. BRASIL. Ministério da Saúde. Secretaria de Vigilância em Saúde. Departamento de Vigilância das Doenças Transmissíveis. Diretrizes para vigilância, atenção e eliminação da Hanseníase como problema de saúde pública: manual técnico-operacional [recurso eletrônico] / Ministério da Saúde, Secretaria de Vigilância em Saúde, Departamento de Vigilância das Doenças Transmissíveis. - Brasília: Ministério da Saúde, 2016; 58 p.: il.

7. CHAVES EC, et al. Índice de carência social e hanseníase no estado do Pará em 2013: análise espacial. Epidemiol e Serv saude Rev do Sist Unico Saude do Bras., 2017; 26(4): 807-16.

8. COSTA LA, et al. Análise epidemiológica da hanseníase na Microrregião de Tucuruí, Amazônia brasileira, com alto percentual de incapacidade física e de casos entre jovens. Rev Pan-Amaz Saúde, 2017; 8(3): 9-17.

9. CUNHA MHCM, et al. Fatores de risco em contatos intradomiciliares de pacientes com hanseníase utilizando variáveis clínicas, sociodemográficas e laboratoriais. Rev Pan-Amaz Saúde, 2017; 8(2): 23-30.

10. DA SILVA JM. Dinâmica territorial da mineração na Mesorregião Sudeste do Estado do Pará - Região Norte do Brasil, 2016.

11. IBGE. INSTITUTO BRASILEIRO DE GEOGRAFIA E ESTATÍSTICA. Divisão Estatística dos estados em Mesorregiões e Microrregiões, 2012.

12. IBGE. INSTITUTO BRASILEIRO DE GEOGRAFIA E ESTATÍSTICA. Estatísticas de gênero. Notas técnicas. Fundamento legal e sigilo das informações, 2014.

13. LOBATO DC, et al. Avaliação das ações da vigilância de contatos domiciliares de pacientes com hanseníase no Município de Igarapé-Açu, Estado do Pará, Brasil. Rev. Pan-Amazônica Saúde, 2016; 7(1): 9-9.

14. MONTEIRO LD, et al. Padrões espaciais da hanseníase em um estado hiperendêmico no Norte do Brasil, 2001 2012. Rev. Saúde Pública, 2015; 49: 84.

15. OLIVEIRA AEVM, et al. Análise epidemiológica da hanseníase por sexo no estado da Paraíba. Research, Society and Development, 2020; 9(8): e755985778.

16. OMS. ORGANIZAÇÃO MUNDIAL SA SAÚDE. Global leprosy update, 2018: moving towards a leprosy free world. Weekly epidemiological record, Genebra, 2019; 94: 389-412.

17. OMS. ORGANIZAÇÃO MUNDIAL DA SAÚDE. Global leprosy update, 2015: time for action, accountability and inclusion. Weekly Epidemiol Rec., 2017; 35 (91): 405 - 420.

18. ONU. ORGANIZAÇÃO DAS NAÇÕES UNIDAS. Objetivos de Desenvolvimento Sustentável até 2030. Nova lorque, 2015.

19. PEREIRA WMM. Distribuição espacial da hanseníase: uma questão sociopolítica. Tese (Doutorado em Biologia Parasitária na Amazônia) - Centro de Ciências Biológicas e da Saúde, Universidade do Estado do Pará/ Instituto Evandro Chagas - Belém, Pará. 2018; 199 p.

20. PASSOS ALV, ARAÚJO LF. Representações sociais da hanseníase: um estudo psicossocial com moradores de um antigo hospital colônia. Interações, Campo Grande, MS, 2020, 21(1): 93-105.

21. PIRES CAA, et al. Análise do perfil clínico- epidemiológico da hanseníase no Pará e avaliação dos indicadores de saúde. Revista Eletrônica Acervo Saúde, 2015; Vol.Sup.27: 1-11.

22. QUARESMA MSM, et al. Perfil clínico e epidemiológico dos pacientes portadores de hanseníase em uma unidade de referência no estado do Pará. Revista Eletrônica Acervo Saúde, 2019; 18: e269-e269.

23. RIBEIRO MDA, et al. Epidemiologic study of leprosy in Brazil: reflections on elimination goals. Rev Panam Salud Publica/Pan Am J Public Heal, 2018; 42: 1-7.

24. RODRIGUES RN, et al. Áreas de alto risco de hanseníase no Brasil, período 2001-2015. Rev Bras Enferm. 2020; 73(3): e20180583.

25. SANTOS DS, et al. Chemoprophylaxis of leprosy with rifampicin in contacts of multibacillary patients: study protocol for a randomized controlled trial. Trials. 2018; 19(1): 244-53.

26. SESPA. SECRETARIA DE ESTADO DE SAÚDE PÚBLICA DO PARÁ. Diretoria de Vigilância Epidemiológica/ Departamento de Epidemiologia. Banco de dados de hanseníase do SINAN, 2004 a 2018.

27. STIVANIN JJ, et al. Casos de hanseníase em cascavel de 2016 a 2018. Revista Thêma et Scientia, $2020 ; 10: 1$. 